\title{
Variables académicas y sociodemográficas relacionadas con el Síndrome de Burnout, en estudiantes de Ingenierías y Ciencias de la Salud de una universidad estatal de Colombia*
}

\section{Academic and demographic variables related to Burnout syndrome in engineering and health sciences programs students of universidad estatal from Colombia}

Recibido: 23 de junio de 2016/Aceptado: 10 de enero de 2017 http://doi.org/10.17081/psico.20.38.2555

\author{
Fernando Robert Ferrel Ortega ${ }^{1}$, Lucía Fernanda Ferrel Ballestas ${ }^{2}$ \\ Universidad Cooperativa de Colombia Sede Santa Marta, Colombia \\ Ada Amalia Cantillo Aguirre ${ }^{3}$, Juliana Jaramillo Campo ${ }^{4}$, Sandra Milena Jiménez Suárez ${ }^{5}$ \\ Universidad del Magdalena, Colombia
}

Palabras clave:

Síndrome de Burnout Académico, Promedio académico, Autoeficacia, Cinismo, Agotamiento emocional.

Key words:

Academic burnout syndrome, GPA, Self-efficacy, Cynicism, Emotional exhaustion.

\begin{abstract}
Resumen
Objetivo: Comparar las dimensiones del Síndrome de Burnout en estudiantes de Ingenierías y Ciencias de la Salud, en relación con las variables académicas (promedio ponderado, semestre y programa cursado) y sociodemográficas (edad, género) de los participantes. Método: Dicho estudio se abordó bajo el enfoque de Bandura (1989), referido a la autoeficacia, bajo el modelo del espiral negativo "hacia abajo", desarrollado por Salanova, Bresó y Schaufeli (2005). Para ello, se desarrolló una investigación de paradigma empírico-analítico, de tipo descriptivo comparativo y diseño transversal. La población estuvo constituida por 1.028 estudiantes de los cuales se obtuvo una muestra estratificada de 280 de ambas facultades participantes; con un porcentaje de error del $5 \%$ y un nivel de confianza del $95 \%$. A ellos se les aplicó el Inventario de Burnout Académico (MBI-SS), validado para este estudio. Luego de obtener los resultados de la prueba, se utilizó el estadístico ANOVA para el respectivo análisis de varianzas por grupos. Resultados: Indicaron un nivel bajo y medio de agotamiento emocional y cinismo, pero con un nivel alto de autoeficacia, con diferencias significativas en autoeficacia con respecto al promedio académico y semestre, y de agotamiento con respecto al semestre, programa y facultad; siendo los estudiantes de Odontología, Medicina, Ingeniería Ambiental y de Sistemas quienes presentaron mayor agotamiento emocional, físico y mental que los otros. Se concluye que existen grupos de estudiantes con diferentes niveles de agotamiento emocional y cinismo, según el género y la carrera que cursan.

Abstract

Objective: Comparison of the Burnout syndrome approach, taking into account academic variables such as: average, semester and program completion and also demographic variables such as: age and gender in students in the engineering and health sciences programs. This study is centered on self-efficacy according to Bandura's theory (1989) and the "downward negative spiral" model developed by Salanova, Bresó y Schaufeli (2005). For this purpose, a paradigm of empirical comparative descriptive and cross sectional research is developed. 1,028 students Method: 280 students were a stratified sample; who belong to the two participant programs; $5 \%$ percent level (the margin of error) with $95 \%$ percent level (a confidence level ). Academic Burnout Syndrome inventory (MBI-SS) was applied to those students, and it was validated for this study. ANOVA statistical test for the respective variance analysis by groups was used in order to get the results. Results: results showed a low and medium level of emotional exhaustion and cynicism but a high level of self-efficacy with significant differences in relation to semester, grade average, exhausting semester, program and faculty were evident. Students from dentistry, medicine, environmental engineering and system program and faculty showed greater physical and mental - emotional exhaustion than the others. It is concluded that emotional exhaustion and cynicism level is different according to gender and career path among the student groups.
\end{abstract}

Referencia de este artículo (APA):

Ferrel, F., Ferrel, L., Cantillo, A., Jaramillo, J. \& Jiménez, S. (2017). Variables académicas y sociodemográficas relacionadas con el Síndrome de Burnout, en estudiantes de Ingenierías y Ciencias de la Salud de una universidad estatal de Colombia. Psicogente, 20(38), 336-352. http://doi.org/10.17081/psico.20.38.2555

* Artículo científico derivado de trabajo de investigación de titulación, Programa de Psicología, Universidad del Magdalena, Colombia, 2014

1. Doctor en Ciencias de la Educación, Docente Investiqador. T. C., Facultad de Psicología. Universidad Cooperativa de Colombia Sede Santa Marta. Email: roferrel@yahoo.com. http://orcid.org/0000-0001-7411-3516

2. Doctora en Ciencias de la Educación, Catedrática y Coordinadora de Investigaciones, T.C., Facultad de Medicina. Universidad Cooperativa de Colombia Sede Santa Marta. Email: lucaferrel@hotmail.com. http://orcid.org/0000-0003-1802-6404

3. Psicóloga. Universidad del Magdalena. Email: adaca08@gmail.com; http://orcid.org/0000-0003-1225-9178

4. Psicóloga. Universidad del Magdalena. Email: juliana.jaramillo@gmail.com; https:/\%orcid.org/0000-0003-0008-9984

5. Psicóloga. Universidad del Magdalena. Email: sami-26j@hotmail.com; https://orcid.org/0000-0002-9265-0937 


\section{Introducción}

En el contexto educativo nacional e internacional se ha puesto de relieve la preocupación por una educación de calidad en todos los niveles escolares, por lo que la investigación de todos aquellos factores, como los psicológicos, que pueden explicarla o mejorarla, son de gran importancia en la actualidad. La necesidad de mantener niveles altos de rendimiento académico dentro de las instituciones de educación superior muchas veces riñe con el bienestar psicológico que deben tener los estudiantes, quienes están constantemente expuestos a sobrecarga de actividades, tareas escolares, falta de espacios de recreación, problemas familiares y económicos, entre otros (Garcés de Los Fayos, 1995; Caballero, Abello y Palacio, 2007).

Todo lo anterior puede llegar a generar un estrés percibido con relación a la actividad estudiantil, que a su vez comienza a predecir índices de agotamiento que tienden a bajar la autoeficacia y configurar el Síndrome de Burnout Académico (SBA). Dicho Síndrome puede comenzar a afectar seriamente el proceso de aprendizaje y la respuesta a los altos estándares académicos propuestos, una vez que sus dimensiones (agotamiento emocional, cinismo e ineficacia) han hecho brote en el individuo, llegando a dañar sus relaciones interpersonales y a afectar su rendimiento académico aumentando el riesgo de adquirir conductas adictivas, generar bajos niveles de satisfacción personal y presentar somatización física como dolor de cabeza, fatiga crónica, cambios en la alimentación y en el sueño, etc. (Barco, Miranda, Herrera y Álvarez, 2008).

Una vez se configura, el SBA se presenta en la sociedad como una problemática de salud pública, pues puede llegar a afectar a los estudiantes en formación y a los nuevos profesionales en ejercicio, quienes, al interactuar con las múltiples obligaciones de su medio académico o laboral, mostrarán un pobre desempeño.

Existen diversas explicaciones teóricas del SBA, una de ellas es la teoría cognitiva social del yo, desarrollada por Bandura (1989), la cual postula que: 1) Las cogniciones influyen en la forma de percibir la realidad, pero a la vez estas cogniciones se modifican por sus efectos y consecuencias observadas. 2) El empeño para la consecución de objetivos y las consecuencias emocionales de las acciones están determinadas por la autoconfianza del sujeto, expresada en variables del self como ser, autoconfianza, autoeficacia y autoconcepto.

Estos mecanismos psicológicos que ayudan a la ejecución de la acción y del pensamiento autorreferente del sujeto, se ven modificados por sus acciones y acumulación de aquellas conductas observadas en los demás, por lo que la creencia o grado de certeza por parte de un sujeto en sus propias capacidades determinará el empeño que pondrá para conseguir sus objetivos y la facilidad o dificultad en conseguirlos. También determinará ciertas reacciones emocionales como la depresión o el estrés. De acuerdo con ello, se incorpora el concepto de "autoeficacia percibida" de Bandura, entendida como las creencias que las personas incorporan acerca de sus capacidades para mantener el control sobre las situaciones que les afectan, y por tanto el éxito y el logro de los objetivos personales obtenidos por uno mismo aumentan los sentimientos de autoeficacia, mientras que el fallo en alcanzar los objetivos conlleva sentimientos de fracaso, baja autoeficacia y puede dar lugar al desarrollo del Síndrome de Burnout.

En este sentido, Bandura (1989) define la autoeficacia como la creencia que se tiene de ser capaz de orga- 
nizar y llevar a cabo actividades que requieren de logro en determinadas situaciones. Luego, en 1997, Bandura describe la competencia percibida y cómo esta influye en la autoeficacia, aclarando que son diferentes, puesto que se refiere a percepciones de eficacia pero en momentos distintos, y señala que las experiencias de éxito, los estados fisiológicos, entre otros, generan mayor autoeficacia positiva y conllevan a éxitos futuros; por el contrario, experimentar el fracaso de manera constante aumenta la evaluación negativa de las competencias, lo cual puede llevar a más fracasos en el futuro.

Por otra parte, el modelo del espiral negativo "hacia abajo", propuesto por Salanova, Bresó y Schaufeli (2005), sugiere que el síndrome de Burnout se desarrolla por la pérdida de energía que experimentan las personas como consecuencia de la demanda de actividad académica y laboral, destacando que la causa principal del Síndrome es la ausencia de eficacia, es decir, la pérdida de expectativas laborales o académicas (Schaufeli, Salanova, GonzálezRomá \& Bakker, 2002), lo que aumenta las creencias de incompetencia y desconfianza, y genera, de esta forma, un espiral negativo. Bajo este modelo, las dimensiones centrales, o 'corazón' del Burnout según sus autores, son el agotamiento y el cinismo, pues consideran que el llamado 'tercer componente' del Burnout, la autoeficacia profesional, ha recibido críticas en muchos sentidos. Por ejemplo, que la autoeficacia profesional es un constructo cercano a una dimensión más estable de la personalidad (Cordes \& Gougherty, 1993; Shirom, 1989) y no tanto un componente central del Burnout, por lo que desempeñaría otro papel profesional (Lee \& Ashforth, 1996). Por otro lado, señalan que algunos teóricos afirman que el desarrollo del Burnout es aquel sentimiento de ineficacia o crisis de autoeficacia que consiste en la falta de confianza en las propias competencias (Cherniss, 1993). Además, destacan que Leiter (1992) retomó la crisis de autoeficacia como el factor esencial para el desarrollo del Síndrome. Finalmente, estos autores postulan que el Burnout se relaciona con la incompetencia personal, profesional y colectiva, que conlleva a creencias negativas debidas al fracaso y la falta de dominio en actividades académicas (Salanova, Llorens, Cifre, Martínez \& Schaufeli, 2003).

Los estudios realizados en distintos contextos, en su mayoría en Estados Unidos de Norteamérica y España, pero también en Latinoamérica, destacan la importancia del SBA como factor de riesgo para el desempeño académico y social en el contexto educativo, algunos de los cuales se presentan a continuación.

Dos estudios en Estados Unidos destacan la presencia del SBA relacionado con variables académicas y de la vida personal en estudiantes de Medicina. Drybye et al (2006) hallaron presencia del SBA en 239 (45\%) estudiantes, con una frecuencia de agotamiento elevada, mientras que la frecuencia de depresión positiva y el consumo de alcohol se halló en riesgo mínimo en estudiantes mayores, y el número de eventos negativos de la vida personal en los últimos 12 meses correlacionó con el riesgo de agotamiento. Los autores concluyeron que el Burnout puede aumentar por año de escolaridad en los estudiantes de Medicina; que el agotamiento emocional está ligado principalmente al estrés laboral y a los acontecimientos de la vida personal.

Por su parte, Santen, Holt, Kemp \& Hemphill (2010) estudiaron el Burnout y factores que inciden en su desarrollo en una muestra de 249 estudiantes de Medicina de la Universidad de Atlanta. Encontraron un nivel moderado o alto grado de agotamiento en el $21 \%$ de la primera clase del año, $41 \%$ de la segunda, el $43 \%$ de la tercera y en el $31 \%$ de la cuarta clase del año $(P<0,05)$. 
La disminución del soporte, el alto estrés y la falta de control sobre la vida fueron relacionados significativamente con Burnout mediante el análisis multivariado. Concluyeron que el Burnout se desarrolla progresivamente en el transcurso de la educación médica, mientras que un alto nivel de apoyo y bajo nivel de estrés disminuyen su presencia.

Estudios realizados en España muestran el impacto negativo del SBA en el desempeño académico y en el bienestar psicológico de los estudiantes universitarios. Así, Bresó y Salanova (2001) llevaron a cabo una investigación en Castellón (España) con el fin de analizar el bienestar psicológico (satisfacción y compromiso académico) de los estudiantes de la UJI y explorar si existían diferencias en función del Centro o Facultad en el que estudian. La muestra fue de 872 estudiantes, de los cuales 302 (34,6 \%) eran de la Escuela Superior de Tecnología y Ciencias Experimentales (ESTCE), 276 (31,7 \%) de la Facultad de Ciencias Jurídico-Económicas (FCJE) y 294 (33,7 \%) de la Facultad de Ciencias Humanas y Sociales (FCHS). Los resultados señalan diferencias según la Facultad en la que estudian: los estudiantes de la FCHS presentaron mayores niveles de satisfacción general y con sus estudios; los de la FCJE presentaron menores niveles de satisfacción total con los estudios y con la Facultad; y los de la ESTCE presentaron un patrón mixto (están más satisfechos con la Facultad, pero no con sus estudios).

En la misma línea, Martínez \& Salanova (2003) realizaron un estudio con el objetivo de analizar el Burnout y el engagement con variables demográficas, desempeño y desarrollo profesional, en una muestra de 525 estudiantes de Psicología, Ingeniería, Técnica Informática y Diplomado en Turismo de la Universita Jaume I de Castellón (España). Los resultados indicaron la exis- tencia de diferencias significativas en las dimensiones de Burnout y engagement en función de la edad, el sexo y la carrera que estudian; y señalan posibles predictores del desempeño y el desarrollo profesional.

En otro estudio realizado en la Universidad de Murcia (España), llamado "Bienestar psicológico en estudiantes universitarios: facilitadores y obstaculizadores del desempeño académico", se hallaron relaciones significativas entre el bienestar psicológico y el rendimiento académico. Se demostró que a mayor rendimiento académico, menor agotamiento; así como a mayor eficacia y vigor, mayor dedicación, mayor autoeficacia, mayor satisfacción y más sensación de felicidad con respecto a los estudios (Salanova, Martínez, Bresó, Llorens y Grau, 2005).

Por otro lado, Preciado y Vázquez (2010), en México, realizaron una investigación sobre perfil de estrés y Síndrome de Burnout en 60 estudiantes (46 \% mujeres y $53 \%$ hombres) de Odontología de una universidad estatal, con promedio de edad 20,65 años. Se encontró que el $27 \%$ presentaba cansancio emocional, el $37 \%$ despersonalización y el 50 \% baja realización personal. El análisis del modelo de regresión estadístico determinó que la débil fuerza cognitiva, las situaciones estresantes, la valoración negativa y el comportamiento tipo A son predictores del Síndrome de Burnout, en tanto que tener buenas redes de apoyo social y bienestar psicológico son variables inversamente correlacionadas con esta dolencia.

Carlotto, Goncalvez y Borges (2005) llevaron a cabo una interesante investigación en Brasil acerca de los predictores del síndrome de Burnout en una muestra de 255 estudiantes de un curso técnico de Enfermería, en el cual analizaron variables demográficas y escolares 
así como los factores de estrés escolar percibido. Los resultados mostraron que el estrés percibido respecto a conciliar la actividad laboral con el curso, conciliar el estudio con las actividades de ocio, realizar exámenes y trabajos, así como la edad, el nivel de expectativas y tener una actividad profesional predicen los índices de agotamiento; que la realización de pruebas y trabajos, la falta de retroalimentación positiva y el estrés percibido para conciliar estudio y familia predicen los niveles de competencia percibidos; y que el predictor del cinismo es el estrés percibido en relación con la falta de retroalimentación positiva y en la relación con los colegas.

En Argentina, Bittar (2008) hizo una investigación sobre el Burnout y los estilos de personalidad en una muestra de 114 estudiantes universitarios de la ciudad de Mendoza. El objetivo del estudio fue analizar si los estudiantes universitarios que trabajan desarrollan el Síndrome de Burnout, y en qué medida los estilos de personalidad pueden incidir en su aparición. Encontró que los estudiantes universitarios que trabajan desarrollan el Síndrome de Burnout y que, si bien los perfiles de personalidad no presentan diferencias significativas entre el grupo que padece el Síndrome y el que no lo presenta, se evidencian rasgos que pueden ser considerados como factores protectores o condicionantes en su aparición.

De igual manera, en Colombia se han venido realizando varios e importantes estudios sobre el SBA. Así, en la costa Caribe se han llevado a cabo una serie de investigaciones con estudiantes universitarios que muestran el impacto del SBA en algunas de sus dimensiones, sin llegar a configurar el síndrome. Caballero, Abello y Palacio (2006) encontraron en estudiantes universitarios de la ciudad de Barranquilla que, aunque no existen diferencias entre las dimensiones del Burnout y el rendimiento académico entre los estudiantes que trabajan y los que no, los estudiantes que laboran y desempeñan más actividades se sienten más autoeficaces. En otra investigación, realizada por los mismos autores en la misma ciudad, sobre la relación del Burnout y el rendimiento académico con la satisfacción frente a los estudios, en una muestra de 202 estudiantes universitarios de la jornada nocturna, encontraron que el 41,6\% presentaron el Síndrome en niveles altos y medio altos, de los cuales el 48,6\% se siente ineficaz, el 38,2\% experimenta agotamiento y el $27,7 \%$ presenta cinismo, aspectos que influirían en el bajo rendimiento académico por la falta de satisfacción (Caballero, Abello y Palacio, 2007). De modo similar, en una investigación realizada sobre el síndrome de burnout en estudiantes de Internado de Medicina, se identificó la presencia del Síndrome en el 9,1\%, en quienes se acentuó un alto grado de agotamiento emocional de un 41,8 \% (Borda et al, 2007). Consecuentemente, se resalta, como respuesta frente a las responsabilidades académicas en presencia del Síndrome de Burnout, una baja percepción de eficacia, desempeño académico bajo y tendencia o riesgo a renunciar del pregrado, caracterizado por promedio deficiente y pérdida de semestres y asignaturas (Caballero, Hederich y Palacio, 2012).

En otro contexto poblacional, se puede citar la investigación sobre el SBA en una muestra de 225 docentes (tiempo completo, parcial y por horas) de una universidad estatal de la ciudad de Santa Marta, realizada por Ferrel, Pedraza y Rubio (2010). Teniendo en cuenta la edad y el tipo de vinculación, encontraron una puntuación baja en las tres dimensiones del Inventario de Burnout Academia (MBI) en los tres tipo de docentes, por lo que se estableció la no presencia de este Síndrome en los docentes y se concluyó, sin embargo, que aunque no se evidencia presencia del Síndrome, hay una tendencia a presentarlo, especialmente en los docentes 
contratados por horas, infiriendo que puede deberse a la sobrecarga de horas y la falta de estabilidad laboral.

Ante este panorama de factores psicológicos que afectan el proceso educativo, como factores de riesgo o protectores, que pueden generar agotamiento y bajo rendimiento o bienestar, satisfacción y alto rendimiento académico en estudiantes de dos de las facultades tradicionalmente identificadas como las de mayor exigencia y compromiso académico por tratarse de ciencias exactas (Ingenierías y Ciencias de la Salud), se desarrolló esta investigación con el objetivo de responder si algunas de las variables académicas y sociodemográficas presentes en la vida del estudiante universitario se relacionan con la presencia del SBA.

\section{Método}

\section{Diseño de investigación}

Esta investigación se realizó desde el paradigma epistemológico empírico-analítico (cuantitativo), de tipo descriptivo-comparativo, consistente en describir el fenómeno de estudio tal como es y se manifiesta, especificando sus propiedades, características y los diversos datos que se recolectan (Hernández, Fernández y Baptista, 2010). En este caso, se compararon las dimensiones del Síndrome de Burnout con variables académicas y sociodemográficas. Se utilizó un diseño transversal, a partir del cual se recolectó la información en un momento único del tiempo, describiendo las variables y su incidencia en un momento dado (Hernández, Fernández y Baptista, 2010).

\section{Participantes}

Se tuvo como población a 1.028 estudiantes: 480 de Ciencias de la Salud (185 de Psicología, 143 de Medicina y 152 de Odontología) y 548 de Ingenierías (102 de Ambiental, 108 de Civil, 105 de Sistemas, 69 de Electrónica y 164 de Industrial); de la Institución participante. De ella, se obtuvo una muestra estratificada de 280 estudiantes de los semestres 2, 5 y 9 con un porcentaje de error del $5 \%$ y un nivel de confianza del $95 \%$; pero, finalmente, se contó con la participación de 254, una pérdida del 9,28\% de la muestra. Como características sociodemográficas se tuvo: $58 \%$ varones y $42 \%$ mujeres; con edades entre 21 y 25 años de edad (52\% del total), entre 16 y 20 años (39\% del total) y entre 26 y 30 años (8\% del total), siendo el rango de menor representatividad el que corresponde a mayores de 31 años.

\section{Instrumentos}

Se utilizó el Inventario de Burnout Académico -MBI-SS (Schaufeli, Martínez, Marques, Salanova \& Bakker, 2002), adaptado de la traducción del Maslach Burnout Inventory-MBI-GS, elaborado por Maslach \& Jackson (1981), el cual evalúa a través de 15 ítems las dimensiones de agotamiento emocional y cinismo que se definen como el corazón del Burnout. En Agotamiento emocional (EX): evalúa la vivencia o sentimiento de encontrarse física, mental y emocionalmente exhausto, y una sensación de no poder dar más de sí mismo frente a las actividades del proceso formativo. Consta de 5 ítems. En Cinismo (CY): evalúa la actitud negativa del estudiante frente a sus estudios, evidenciada a través de la autocrítica, desvalorización, pérdida del interés, la trascendencia y valor frente al estudio. Consta de 5 ítems. En Eficacia académica (PEF): evalúa la percepción del estudiante sobre la competencia en sus estudios y comprende 5 ítems. La calificación comprende cuatro categorías: Bajo (8 a 15 puntos), Medio Bajo (15 a 19 
puntos), Medio Alto (19 a 24 puntos) y Alto (más de 24 puntos). Este instrumento fue validado para la presente investigación con una muestra de estudiantes universitarios de la ciudad de Santa Marta, mostrando una confiabilidad satisfactoria por medio del Alfa de Cronbach en las tres escalas: Agotamiento emocional $\alpha=0,832$; Cinismo $\alpha=0,809$ y Eficacia académica $\alpha=0,747$. Este Instrumento posee una validez de constructo obtenido mediante análisis factorial (Silvia, 2009).

\section{Análisis de los datos}

Los datos obtenidos fueron procesados mediante el software SPSS v19.0 y para su interpretación se utilizó el estadístico ANOVA para el respectivo análisis de varianzas. Los resultados encontrados fueron tratados garantizando los aspectos éticos contenidos en la Resolución 8430 de 1993 del Ministerio de Salud, por la cual se establecen las normas científicas, técnicas y administrativas para la investigación en salud mental. También, los aspectos éticos contenidos en la Ley 1090 de 2006 o Ley del Psicólogo en Colombia, en la cual se contempla el derecho a la confiabilidad de la información obtenida de la persona en el desarrollo de un proceso de investigación y el bienestar de los participantes humanos.

\section{Resultados}

A continuación se presentan los resultados de las dimensiones del SBA por niveles, las dimensiones del SBA y las variables académicas, las dimensiones del SBA y las variables sociodemográficas (género y edad), y las diferencias significativas entre las dimensiones del SBA con las variables académicas y sociodemográficas. Dichos resultados permitirán describir los hallazgos significativos encontrados en esta investigación.
Tabla 1. Resultados generales del SBA, por niveles

\begin{tabular}{lccc}
\hline Dimensiones & Niveles & Frecuencia & Porcentaje \\
\hline \multirow{4}{*}{ Agotamiento } & Nivel Bajo & 121 & 47,6 \\
& Nivel Medio & 108 & 42,5 \\
& Nivel Alto & 25 & 9,8 \\
& Total & 254 & 100 \\
& Niveles & Frecuencia & Porcentaje \\
& Nivel Bajo & 235 & 92,5 \\
Cinismo & Niveló Medio & 12 & 4,7 \\
& Nivel Alto & 7 & 2,8 \\
& Total & 254 & 100 \\
Autoeficacia & Nivel Bajo & 4 & 1,6 \\
& Nivel Medio & 82 & 32,3 \\
Total & Nivel Alto & 168 & 66,1 \\
\hline
\end{tabular}

Los resultados generales hallados sobre las diferentes dimensiones del SB indican que el 47,6 \% de los estudiantes presentaron un nivel bajo en la dimensión agotamiento emocional, el 42,5\% presentaron un nivel medio y el 9,8\% presentaron un nivel alto. Los anteriores resultados indican que más de la mitad de los estudiantes experimentan la vivencia o sentimiento de encontrarse física, mental y emocionalmente exhaustos y una sensación de no poder dar más de sí mismos frente a las actividades del proceso formativo. Respecto a la dimensión cinismo, la mayoría de los estudiantes $(92,5 \%)$ presentaron un nivel bajo, solo el 4,7 \% presentó un nivel medio y el 2,8 \% nivel alto, lo cual indica que una pequeña proporción de estudiantes (7,5\%) experimentan aspectos de autocritica, desvalorización y pérdida del interés, de la trascendencia y valor frente al estudio. Finalmente, en relación con la eficiencia académica, se encontró que el 66,1 \% presenta un nivel alto, el 32,3 \% un nivel medio y solo el 1,6 \% presentó un nivel bajo, lo cual indica que más de la mitad de los participantes consideran que se desarrollan de manera eficaz en relación a sus actividades académicas, 
se sienten estimulados a lograr sus objetivos académicos y se consideran buenos estudiantes, entre otros aspectos (Tabla 1).

Tabla 2. Distribución por Dimensiones del SBA con relación a la variable Promedio Académico

\begin{tabular}{|c|c|c|c|c|c|c|}
\hline $\begin{array}{l}\text { Dimensiones } \\
\text { del Síndrome } \\
\text { Burnout }\end{array}$ & $\begin{array}{l}\text { Variable } \\
\text { académica } \\
\text { Promedio } \\
\text { académico }\end{array}$ & $\mathrm{N}$ & Media & $\begin{array}{l}\text { Desviación } \\
\text { típica }\end{array}$ & $\mathrm{F}$ & Sig. \\
\hline \multirow[t]{4}{*}{ Agotamiento } & $\begin{array}{l}\text { Nivel bajo } \\
(320-379)\end{array}$ & 164 & 12,5793 & 6,18129 & 1,481 & 0,229 \\
\hline & $\begin{array}{l}\text { Nivel medio } \\
\text { (380.424) }\end{array}$ & 80 & 11,9625 & 5,70686 & & \\
\hline & $\begin{array}{l}\text { Nivel alto } \\
(425-500)\end{array}$ & 10 & 9,4 & 4,06065 & & \\
\hline & Total & 254 & 12,2598 & 5,98247 & & \\
\hline \multirow[t]{4}{*}{ Cinismo } & $\begin{array}{l}\text { Nivel bajo } \\
(320-379)\end{array}$ & 164 & 2,7988 & 3,82305 & 1,306 & 0,273 \\
\hline & $\begin{array}{l}\text { Nivel medio } \\
(380-424)\end{array}$ & 80 & 2,1625 & 3,11568 & & \\
\hline & $\begin{array}{l}\text { Nivel alto } \\
(425-500)\end{array}$ & 10 & 1,5 & 2,12132 & & \\
\hline & Total & 254 & 2,5472 & 3,56915 & & \\
\hline \multirow[t]{4}{*}{ Autoeficacia } & $\begin{array}{l}\text { Nivel bajo } \\
(320-379)\end{array}$ & 164 & 24,9329 & 5,13873 & 5,913 & $0,003^{*}$ \\
\hline & $\begin{array}{l}\text { Nivel medio } \\
(380-424)\end{array}$ & 80 & 27,05 & 4,28096 & & \\
\hline & $\begin{array}{l}\text { Nivel alto } \\
(425-500)\end{array}$ & 10 & 27,6 & 4,16867 & & \\
\hline & Total & 254 & 25,7047 & 4,9451 & & \\
\hline
\end{tabular}

* Diferencias significativas al 0,05

Se resalta que no se hallaron diferencias significativas entre el promedio académico de los estudiantes y las dimensiones de agotamiento $(F=1,481 ; \mathrm{p}<0,229)$ ni de cinismo $(F=1,306 ; p<0,273)$. Sin embargo, de acuerdo al análisis de media se observó una mayor proporción de agotamiento y cinismo entre los estudiantes que tienen un promedio bajo (320-379). En cuanto a la percepción de autoeficacia académica se hallaron dife- rencias significativas $(F=5,913 ; p>0,003)$ entre los estudiantes que tienen promedio académico alto en comparación con los niveles medios y bajo, lo que sugiere una mayor percepción de autoeficacia en los estudiantes que tienen un promedio académico alto (Tabla 2).

Tabla 3. Distribución por Dimensiones del SBA con relación a la variable Semestre

\begin{tabular}{lllllll}
\hline $\begin{array}{c}\text { Dimensiones } \\
\text { del síndrome } \\
\text { Burnout }\end{array}$ & $\begin{array}{c}\text { Variable } \\
\text { Académica } \\
\text { (Semestre) }\end{array}$ & N & Media & $\begin{array}{c}\text { Desviación } \\
\text { típica }\end{array}$ & F & Sig. \\
\hline Agotamiento & Segundo & 50 & 9,7 & 4,50057 & 6,915 & $0,001^{*}$ \\
Quinto & 90 & 13,5222 & 6,38507 & & & \\
Noveno & 114 & 12,386 & 5,92058 & & & \\
Total & 254 & 12,2598 & 5,98247 & & & \\
Cinismo & Segundo & 50 & 2 & 3,09707 & 1,735 & 0,178 \\
Quinto & 90 & 3,0778 & 4,13053 & & & \\
Noveno & 114 & 2,3684 & 3,24586 & & & \\
Total & 254 & 2,5472 & 3,56915 & & & \\
Autoeficacia & Segundo & 50 & 25,96 & 5,8203 & 3,984 & $0,02^{*}$ \\
Quinto & 90 & 24,5667 & 4,7807 & & & \\
Noveno & 114 & 26,4912 & 4,51398 & & & \\
Total & 254 & 25,7047 & 4,9451 & & & \\
\hline *Diferencias significativa al 0,05 & & & & \\
& & & & & & \\
\hline
\end{tabular}

De igual manera, no se hallaron diferencias significativas entre la dimensión de cinismo con semestre académico $(F=1,735 ; p<0.178)$. Sin embargo, se observó diferencia significativa en los niveles de agotamiento $(F=6,915 ; p>0,001)$ con el quinto semestre en comparación con los de segundo y noveno; es decir, los estudiantes que cursan quinto semestre están más agotados que los de segundo y noveno. De igual manera, se halló diferencia significativa en la autoeficacia académica $(\mathrm{F}=$ 3,984; $\mathrm{p}>0,02$ ) con los estudiantes que cursan noveno semestre en comparación a los de segundo y quinto semestre (Tabla 3). 
Tabla 4. Distribución por Dimensiones del SBA con relación a la variable Programa

\begin{tabular}{|c|c|c|c|c|c|c|}
\hline $\begin{array}{l}\text { Dimensiones } \\
\text { del Síndrome } \\
\text { Burnout }\end{array}$ & $\begin{array}{l}\text { Variable } \\
\text { Académica } \\
\text { de programa }\end{array}$ & $\mathrm{N}$ & Media & $\begin{array}{l}\text { Desviación } \\
\text { típica }\end{array}$ & $\mathrm{F}$ & Sig. \\
\hline \multirow[t]{9}{*}{ Agotamiento } & Psicologia & 50 & 11,3400 & 5,53508 & \multirow[t]{9}{*}{5,593} & \multirow[t]{9}{*}{, 000 } \\
\hline & Medicina & 37 & 12,8649 & 5,86971 & & \\
\hline & Odontología & 41 & 16,6585 & 6,20729 & & \\
\hline & Industrial & 44 & 10,0227 & 5,16468 & & \\
\hline & Electrónica & 18 & 11,0556 & 5,50371 & & \\
\hline & Sistemas & 24 & 12,9167 & 5,85266 & & \\
\hline & Civil & 18 & 9,5000 & 4,30116 & & \\
\hline & Ambiental & 22 & 12,1364 & 6,03399 & & \\
\hline & Total & 254 & 12,2598 & 5,98247 & & \\
\hline \multirow[t]{9}{*}{ Cinismo } & Psicología & 50 & 1,6600 & 2,67727 & \multirow[t]{9}{*}{2,105} & \multirow[t]{9}{*}{,044 } \\
\hline & Medicina & 37 & 2,8378 & 3,73804 & & \\
\hline & Odontología & 41 & 3,4878 & 3,68864 & & \\
\hline & Industrial & 44 & 2,4773 & 3,56657 & & \\
\hline & Electrónica & 18 & 2,2222 & 3,09754 & & \\
\hline & Sistemas & 24 & 3,8333 & 4,55561 & & \\
\hline & Civil & 18 &, 7222 & 1,31978 & & \\
\hline & Ambiental & 22 & 2,8182 & 4,50012 & & \\
\hline & Total & 254 & 2,5472 & 3,56915 & & \\
\hline \multirow[t]{9}{*}{ Autoeficacia } & Psicologia & 50 & 26,5200 & 4,87493 & \multirow[t]{9}{*}{1,450} & \multirow[t]{9}{*}{,186 } \\
\hline & Medicina & 37 & 24,0541 & 6,28466 & & \\
\hline & Odontologia & 41 & 26,8537 & 4,77264 & & \\
\hline & Industrial & 44 & 25,5000 & 4,07773 & & \\
\hline & Electrónica & 18 & 25,8333 & 3,12956 & & \\
\hline & Sistemas & 24 & 24,5833 & 6,01387 & & \\
\hline & Civil & 18 & 26,7222 & 3,84716 & & \\
\hline & Ambiental & 22 & 25,1818 & 4,77729 & & \\
\hline & Total & 254 & 25,7047 & 4,94510 & & \\
\hline
\end{tabular}

${ }^{*}$ Diferencias significativas al 0,05

En cuanto a las diferencias entre Programas Académicos y las dimensiones del SB evaluadas, se encontraron diferencias significativas en el programa de Psicología y las dimensiones agotamiento $(F=5,593 ; p<0,000$, significativo al nivel de $0,05 \%)$ y cinismo $(F=2,105$; $\mathrm{p}<0.044)$. No se encontraron diferencias significativas con la dimensión de cinismo $(\mathrm{F}=2,105 ; \mathrm{p}<0.044)$ y autoeficacia. Sin embargo, se halló que los estudiantes de Odontología tienden a padecer síntomas de agotamiento, pero, igualmente, los estudiantes de Odontología, como los de Psicología, mostraron los mayores niveles de autoeficacia (Tabla 4).

Tabla 5. Distribución por Dimensiones del SBA con relación a la variable Facultad

\begin{tabular}{|c|c|c|c|c|c|c|}
\hline $\begin{array}{l}\text { Dimensiones } \\
\text { del síndrome } \\
\text { Burnout }\end{array}$ & $\begin{array}{l}\text { Variable } \\
\text { Académica } \\
\text { Facultad }\end{array}$ & $\mathrm{N}$ & Media & $\begin{array}{l}\text { Desviación } \\
\text { Típica }\end{array}$ & $\mathrm{F}$ & Sig. \\
\hline \multirow[t]{3}{*}{ Agotamiento } & $\begin{array}{l}\text { Facultad de las } \\
\text { Ciencias de la } \\
\text { Salud }\end{array}$ & 128 & 13,4844 & 6,23678 & 11,249 &, $001^{*}$ \\
\hline & $\begin{array}{l}\text { Facultad de las } \\
\text { Ingenierías }\end{array}$ & 126 & 11,0159 & 5,46111 & & \\
\hline & Total & 254 & 12,2598 & 5,98247 & & \\
\hline \multirow[t]{3}{*}{ Cinismo } & $\begin{array}{l}\text { Facultad de las } \\
\text { Ciencias de la } \\
\text { Salud }\end{array}$ & 128 & 2,5859 & 3,41033 & ,030 & 862 \\
\hline & $\begin{array}{l}\text { Facultad de las } \\
\text { Ingenierías }\end{array}$ & 126 & 2,5079 & 3,73684 & & \\
\hline & Total & 254 & 2,5472 & 3,56915 & & \\
\hline \multirow[t]{3}{*}{ Autoeficacia } & $\begin{array}{l}\text { Facultad de las } \\
\text { Ciencias de la } \\
\text { Salud }\end{array}$ & 128 & 25,9141 & 5,38301 & ,461 & ,498 \\
\hline & $\begin{array}{l}\text { Facultad de las } \\
\text { Ingenierías }\end{array}$ & 126 & 25,4921 & 4,4681 & & \\
\hline & Total & 254 & 25,7047 & 4,9451 & & \\
\hline
\end{tabular}

* Diferencias significativas al 0,05

En el mismo sentido, no se hallaron diferencias significativas entre la dimensión cinismo y la variable Facultad $(F=0,030 ; p<0.0862)$, como tampoco entre la dimensión autoeficacia y facultad $(F=0,461 ; p<0.498)$. Sin embargo, con relación al agotamiento, se encontró en un nivel significativo, siendo la Facultad de Ciencias 
de la Salud la que presentó mayor dificultad con relación a esta dimensión (Tabla 5).

Tabla 6. Dimensiones del SBA con relación a la variable Sociodemográfica Género

\begin{tabular}{ccccccc}
\hline $\begin{array}{c}\text { Dimensiones } \\
\text { del síndrome } \\
\text { Burnout }\end{array}$ & $\begin{array}{c}\text { Variable } \\
\text { Socio- } \\
\text { demográfica } \\
\text { Género }\end{array}$ & N & Media & $\begin{array}{c}\text { Desviación } \\
\text { típica }\end{array}$ & $\mathrm{F}$ & Sig. \\
\hline Agotamiento & Mujer & 107 & 11,9626 & 5,95094 &, 455 &, 500 \\
& Hombre & 147 & 12,4762 & 6,01634 & & \\
& Total & 254 & 12,2598 & 5,98247 & & \\
Cinismo & Mujer & 107 & 1,7850 & 2,71970 & 8,688 &, $004^{*}$ \\
& Hombre & 147 & 3,1020 & 3,99612 & & \\
& Total & 254 & 2,5472 & 3,56915 & & \\
Autoeficacia & Mujer & 107 & 26,5888 & 4,58454 & 6,026 &, 015 \\
& Hombre & 147 & 25,0612 & 5,11140 & & \\
& Total & 254 & 25,7047 & 4,94510 & & \\
\hline
\end{tabular}

Diferencias significativa al 0,05

Respecto al género de los estudiantes, se encontró que no existen diferencias significativas entre la variable género y la dimensión de cinismo $(F=8,688$; p < 0.004). Sin embargo, con relación a las dimensiones de agotamiento y autoeficacia se evidenció que sí existen diferencias significativas: $(F=, 455 ; \mathrm{p}>, 500) \mathrm{y}$ $(F=6,026 ; p>, 015)$, respectivamente. Lo anterior indica que tanto en hombres como en mujeres, el nivel de agotamiento emocional es bajo; y que respecto a la dimensión de autoeficacia los dos géneros se encuentran en un nivel alto, siendo más alto en las mujeres con relación a los varones. Respecto a la dimensión cinismo los dos géneros se encuentran en un nivel alto, siendo más alto para las mujeres (Tabla 6).
Tabla 7. Dimensiones del SBA con relación a la variable Sociodemográfica Edad

\begin{tabular}{|c|c|c|c|c|c|c|}
\hline $\begin{array}{l}\text { Dimensiones } \\
\text { del Síndrome } \\
\text { Burnout }\end{array}$ & $\begin{array}{l}\text { Variable } \\
\text { Socio } \\
\text { demográfica } \\
\text { Edad }\end{array}$ & $\mathrm{N}$ & Media & $\begin{array}{l}\text { Desviación } \\
\text { típica }\end{array}$ & $\mathrm{F}$ & Sig. \\
\hline \multirow[t]{4}{*}{ Agotamiento } & 16 A 20 Años & 98 & 12,3673 & 6,16841 & \multirow[t]{4}{*}{1,206} & \multirow[t]{4}{*}{, 301} \\
\hline & 21 A 25 Años & 132 & 11,8788 & 5,43810 & & \\
\hline & 26A 31 Años & 24 & 13,9167 & 7,81257 & & \\
\hline & Total & 254 & 12,2598 & 5,98247 & & \\
\hline \multirow[t]{4}{*}{ Cinismo } & 16 A 20 Años & 98 & 2,2551 & 3,22462 & \multirow[t]{4}{*}{.557} & \multirow[t]{4}{*}{, 574} \\
\hline & 21 A 25 Años & 132 & 2,7576 & 3,74190 & & \\
\hline & 26 A 31 Años & 24 & 2,5833 & 3,98821 & & \\
\hline & Total & 254 & 2,5472 & 3,56915 & & \\
\hline \multirow[t]{4}{*}{ Autoeficacia } & 16 A 20 Años & 98 & 26,0408 & 5,30302 & \multirow[t]{4}{*}{ _, 414} & \multirow[t]{4}{*}{,662 } \\
\hline & 21 A 25 Años & 132 & 25,5455 & 4,46422 & & \\
\hline & 26 A 31 Años & 24 & 25,2083 & 5,99985 & & \\
\hline & Total & 254 & 25,7047 & 4,94510 & & \\
\hline
\end{tabular}

${ }^{*}$ Diferencias significativas al 0,05

Respecto a la edad, no se encontraron diferencias significativas con los niveles de agotamiento $(\mathrm{F}=1,481$; $\mathrm{p}<0,229)$, cinismo $(\mathrm{F}=1,306 ; \mathrm{p}<0,273)$ y autoeficacia $(\mathrm{F}=1,206)$. Sin embargo, se evidenció, con relación al agotamiento emocional, que todas las personas se encuentran en un nivel bajo (Media $=12,25)$, siendo el segmento de las personas que están entre 26 y 31 años quienes tienen el mayor nivel $($ Media $=13,96)$. Con relación a la autoeficacia, el estudio arrojó como resultado que el rango de edades entre 16 y 20 años tiene el nivel más alto $($ Media $=26,04)($ Tabla 7$)$.

Finalmente, respecto a la relación entre el SB y las variables académicas, se encontró diferencia significativa entre el agotamiento emocional y las variables académicas como semestres $(\mathrm{F}=6,915 ; \mathrm{P}>0,001)$, programas académicos $(F=5,593 ; P>0,00)$ y Facultad $(F=11,249$; $\mathrm{P}>0,001)$. En igual sentido, se hallaron diferencias sig- 
nificativas entre la autoeficacia y los promedios académicos $(F=5,913 ; P>0,003)$ y Semestres $(F=3,984$; P > 0,02). Llaman la atención también las diferencias significativas encontradas respecto a la dimensión de cinismo respecto al género de los estudiantes $(F=8,688$; P > ,004) (Tabla 8).

Tabla 8. Diferencias significativas de las dimensiones del SBA con variables Académicas y Sociodemográficas

\begin{tabular}{|c|c|c|c|c|c|c|}
\hline \multirow{2}{*}{$\begin{array}{l}\text { Dimensiones } \\
\text { del Síndrome } \\
\text { de Burnout }\end{array}$} & \multicolumn{4}{|c|}{ Variables Académicas } & \multicolumn{2}{|c|}{$\begin{array}{c}\text { Variables } \\
\text { Sociodemográficas }\end{array}$} \\
\hline & Promedio & Semestre & Programa & Facultad & Género & Edad \\
\hline \multirow[t]{2}{*}{ Agotamiento } & $\mathrm{F}=1,481$ & $\mathrm{~F}=6,915$ & $\mathrm{~F}=5,593$ & $\mathrm{~F}=11,249$ & $\mathrm{~F}=455$ & $F=1,206$ \\
\hline & $P<0,229$ & $P>0,001^{*}$ & $P>0,00^{*}$ & $P>0,001^{*}$ & $\mathrm{P}<500$ & $\mathrm{P}<0,301$ \\
\hline \multirow[t]{2}{*}{ Cinismo } & $\mathrm{F}=1,306$ & $\mathrm{~F}=1,735$ & $\mathrm{~F}=2,105$ & $\mathrm{~F}=0,03$ & $F=8,688$ & $\mathrm{~F}=557$ \\
\hline & $P<0,273$ & $P<0,178$ & $\mathrm{P}<0,044$ & $P<0,862$ & $P>, 004^{*}$ & $\mathrm{P}<, 574$ \\
\hline \multirow[t]{2}{*}{ Autoeficacia } & $\mathrm{F}=5,913$ & $\mathrm{~F}=3,984$ & $\mathrm{~F}=1,45$ & $\mathrm{~F}=0,461$ & $\mathrm{~F}=6,026$ & $\mathrm{~F}=, 414$ \\
\hline & $P>0,003^{*}$ & $P>0,02^{*}$ & $\mathrm{P}<0,186$ & $P<0,498$ & $\mathrm{P}<, 015$ & $\mathrm{P}<, 662$ \\
\hline
\end{tabular}

${ }^{*}$ Diferencias significativa al 0,05

\section{Discusión}

El presente estudio tuvo como objetivo comparar las dimensiones del Síndrome de Burnout en estudiantes de Ingenierías y Ciencias de la Salud, en relación a las variables académicas (promedio ponderado, semestre y programa cursado) y sociodemográficas (edad, género) de los participantes. Los datos encontrados en este estudio no evidencian la presencia del Síndrome, el cual requiere la presencia de altos niveles de agotamiento emocional y cinismo y bajos de autoeficacia, pero sí importantes niveles de sus dimensiones que se relacionan con las variables académicas estudiadas.

La alta concentración en los niveles bajos y medios de agotamiento emocional, los niveles bajos de cinismo y, a pesar de lo anterior, los altos niveles de autoeficacia de los participantes, indican que estos programas acadé- micos poseen porciones de estudiantes que se distribuyen entre no agotados física, mental y emocionalmente, y moderadamente agotados; así como de estudiantes que tienen una buena relación o actitud hacia el trabajo académico, de modo que tienden a experimentar creencias de eficacia ante los estudios.

$\mathrm{Al}$ respecto, los resultados encontrados por Barraza, Carrasco y Arreola (2009), en México, sobre la presencia del Síndrome en un nivel leve, confirman los obtenidos en la presente investigación, en la cual se evidenció que si bien existen algunas características de las dimensiones evaluadas del Síndrome de Burnout, estas no evidencian una presencia representativa en la población participante.

En cuanto a las dimensiones del SBA y las variables académicas (promedio ponderado, semestre, programa académico y facultad), se encontraron diferencias significativas en la dimensión de autoeficacia académica en relación con promedio académico y semestre; y entre agotamiento emocional con semestre, programa y facultad.

Respecto a la autoeficacia, se halló que existen diferencias en la percepción de competencias entre los estudiantes con promedio académico alto en comparación con los de promedios bajos y medios. Así mismo, con los estudiantes que cursan noveno semestre en comparación con los que cursan quinto y segundo semestre. Desde la perspectiva cognitiva social del yo (Bandura, 1989), la autovaloración ayuda a los estudiantes a la ejecución de la acción, mediada por el pensamiento autorreferencial, las cogniciones y creencias o grado de certeza acerca de sus propias capacidades para determinar su desempeño en función de los objetivos y de la facilidad para mantener un promedio académico alto (Ortega y López, 2004). 
Es interesante destacar que existen diferencias en cuanto a la autoeficacia percibida según el semestre cursado. Así, los estudiantes que cursan noveno semestre muestran mayor percepción de autoeficacia en comparación con los de segundo y quinto semestre. Lo anterior indica la importancia de promover logros exitosos en el ejercicio académico desde los primeros semestres de estudio. De acuerdo a la teoría, el éxito, el logro de los objetivos académicos y las consecuentes experiencias positivas obtenidas al cursar hasta el noveno semestre, experimentando sentimientos de éxito, aumentando la autoestima, motivación y satisfacción y sintiéndose preparado para ejercer como profesional, refuerzan la percepción de autoeficacia; mientras que la dificultad en alcanzar los objetivos conlleva sentimientos de fracaso, baja autoeficacia y podría ser un factor de riesgo para el desarrollo del Síndrome de Burnout. Estos resultados son coherentes con los hallados por Schaufeli et al (2002)

En lo que respecta al programa académico cursado, los estudiantes de Odontología, Medicina, Ingeniería Ambiental y de Sistemas presentaron mayor agotamiento físico y mental en comparación con los estudiantes de Psicología, Ingeniería Civil, Industrial y Electrónica. Estos resultados podrían sugerir, de acuerdo a lo planteado por algunos estudios, que la carrera cursada puede ser una variable facilitadora para generar agotamiento físico y mental ante las actividades académicas, lo que se confirma por los resultados planteados por GilMonte y Peiró (1999), los cuales indican que la mayoría de los estudiantes tienden a presentar sentimientos de cansancio físico y psicológico y sensación de agotamiento debido a los requerimientos del estudio (Maslach \& Jackson, 1981; Gil-Monte, 2001), siendo los estudiantes de Odontología y Medicina los más agotados.
De otra parte, se halló también que los estudiantes de Ciencias de la Salud (Odontología y Medicina) presentan más agotamiento que los estudiantes de las Ingenierías (Ingeniería Ambiental y de Sistemas), lo que podría sugerir estos programas como predictores para el agotamiento. Al respecto, diversos autores señalan que los profesionales en formación de atención sanitaria, al estar expuestos a procesos académicos evaluativos en paralelo al afrontamiento con la asistencia en salud, propia de las prácticas formativas en cada una de las disciplinas, demanda en los estudiantes exigencias personales en sus primeras aproximaciones a pacientes y evaluación docente, propiciando individuos más agotados (Drybye et al, 2006; Deary, Watson \& Houston, 2003). Estos resultados son corroborados con los encontrados por Barraza, Ortega y Ortega (2013), en comparación con otras disciplinas como las Ingenierías.

De otra parte, si bien los resultados evidencian que no existen diferencias significativas en las dimensiones de cinismo con las variables académicas, se observó que dicha variable difiere levemente con el semestre, promedio académico, programas y las facultades (Ciencias de la Salud e Ingeniería). Es decir, los estudiantes de quinto semestre tienden a estar más cínicos, autocríticos y con una actitud de indiferencia frente a los estudios, en comparación con los de segundo y noveno semestre. También, los estudiantes de promedio académico bajo son más cínicos en comparación con los de promedio medio y alto. Por tanto, se puede afirmar, en acuerdo con Martínez y Márquez (2005) y Salanova y Schaufeli (2004), que los estudiantes que presentan niveles altos o medios en cinismo también presentan un promedio académico más bajo.

En cuanto al programa académico cursado, los estudiantes pertenecientes a los programas de Ingenie- 
ría de Sistemas, Odontología, Medicina e Ingeniería Ambiental se observan más cínicos, es decir, que están más predispuestos a cambios en actitudes y conductas (afrontamiento defensivo), a un trato frío, impersonal e indiferente con sus docentes y compañeros de estudio en comparación con los estudiantes de Ingeniería Civil, Industrial, Electrónica y Psicología. Y, en lo que respecta a la Facultad, los estudiantes de Ciencias de la Salud muestran más cinismo en comparación con los estudiantes de las Ingenierías, lo cual puede considerarse una debilidad en la formación de los futuros profesionales de la salud que debe afrontarse desde los primeros semestres.

Por otro lado, al comparar las dimensiones del SBA con las variables de género y edad, se encontró que no hay diferencias significativas en la dimensión de agotamiento y autoeficacia. Sin embargo, se hallaron diferencias significativas con las variables cinismo y género, pero no con la variable edad. De esta manera, el género masculino presenta más cinismo, es decir, son más autocríticos, muestran un comportamiento indiferente hacia el compromiso académico y una actitud fría ante sus compañeros, en comparación con el género femenino. Aunque diferentes investigadores señalan que las mujeres son las que tienden a padecer el Síndrome con mayor frecuencia o a desarrollar sus variables, tal como lo afirman Caballero, Hederich y Palacio (2010), en esta investigación los datos encontrados son contrarios. Así mismo, se puede comprobar que algunas investigaciones presentan resultados similares a los de este estudio, como las realizadas por Martínez y Márquez (2005) y Martínez y Salanova (2003). Mientras que en la dimensión cinismo no se encontró diferencia significativa en relación con la variable edad, se observó que dicha dimensión difiere levemente con la edad comprendida entre $21 \mathrm{y}$ 25 años, siendo estos estudiantes los que muestran más cinismo hacia el trabajo, en comparación con los de 26 a
31 años y los de 16 a 20. En este sentido, esta dimensión del Síndrome no está relacionada con los rangos de edad propuestos por los investigadores.

Con relación a la dimensión de agotamiento con el género y la edad, tampoco se hallaron diferencias significativas, si bien se mostró que dicha dimensión difiere levemente con el género, siendo los hombres los que tienden a estar más agotados física, mental y emocionalmente en comparación con las mujeres. Estos resultados no coinciden con lo expuesto por Gil-Monte y Peiró (1999), quienes afirmaron que las mujeres tienden a experimentar más agotamiento emocional y más intenso que los hombres. De igual forma, los hallazgos obtenidos en esta investigación coinciden con los encontrados por Martínez y Márquez (2005). Del mismo modo, existe una tendencia a que en la dimensión de agotamiento la media se eleve entre los 26 y los 31 años de edad; probablemente esta porción de personas, además de dedicarse a las actividades académicas, mantienen una vida activa laboralmente y poseen responsabilidades familiares.

En cuanto a la dimensión de autoeficacia, no se observaron diferencias significativas con el género y la edad, pero se encontró una leve diferencia en las mujeres, siendo estas más autoeficaces, mostrando mejor desempeño y mejores expectativas de éxito. Estos resultados coinciden con los obtenidos por Martínez y Salanova (2003) y Salanova, Martínez, Bresó, Llorente y Grau (2005), en los que las mujeres se percibían más autoeficaces en comparación con los hombres. Por otro lado, encontramos que otro estudio contradice estos resultados en los cuales las mujeres suelen ser menos autoeficaces que los hombres, (Caballero, Hederich y Palacio, 2010).

Al igual que el género, la variable edad con la 
dimensión de agotamiento no muestra diferencias significativas, pero sí se encontró una leve diferencia en las edades comprendidas entre 16 y 20 años y con las edades de 21 a 25 y 26 a 31 años. Con respecto a esto, Gil-Monte y Peiró (1999) resaltan que las variables sociodemográficas del Burnout deben considerarse como inconsistentes; aunque en otros hallazgos se puede confirmar lo encontrado en nuestro estudio.

En conclusión, la dimensión de agotamiento se diferenció significativamente en variables académicas como semestres, programas académicos y facultad, siendo el semestre el que se relaciona con más agotamiento. En lo que respecta a Programas: Odontología, Sistemas, Medicina y Ambiental son los que tienden a presentar mayor agotamiento. En cuanto a los estudiantes, los que pertenecen a Ciencias de la Salud son los que mostraron mayor agotamiento. Por su parte, la autoeficacia encontró diferencias significativas en cuanto a promedio de calificaciones y semestres, mostrando mayor eficacia los estudiantes con promedios altos y los que cursaban noveno semestre. Y, en cuanto al cinismo, se diferenció estadísticamente en el género masculino.

Teóricamente, los autores sugieren una explicación desde la teoría del "espiral negativo-hacia abajo", desarrollado por Salanova, Bresó y Schaufeli (2005), en el que los estudiantes que presentaron mayor rendimiento frente a sus compromisos académicos manifestaron mayores niveles de autoeficacia en comparación con aquellos que tienen niveles inferiores de rendimiento académico, ya que los primeros manejan creencias positivas sobre sí, como son: la capacidad de organizarse y realizar actividades que concreten sus logros, lo que permite tener una percepción de mayor competencia que influye favorablemente en la autoeficacia y, por tanto, en los éxitos del futuro.
Finalmente, en tanto que el presente estudio permitió reconocer la existencia en nivel bajo de algunas dimensiones del Síndrome de Burnout en conglomerados de estudiantes, se recomienda la atención preventiva de la institución universitaria frente a este Síndrome, para un manejo adecuado del problema y la promoción de estilos de vida saludables en la población universitaria, que puedan facilitar su proceso de formación académica. Igualmente, se recomienda continuar con otros estudios en carreras de Ciencias Sociales y Humanas, como las de Derecho o Educación, que incluyen una práctica social pre-profesional en su pensum de estudios, en la que tienen que atender a población necesitada que le exige un contacto y compromiso permanentes. En estos futuros estudios se recomienda incluir otras variables individuales, como las de personalidad o estilos de vida, grupales y organizacionales (Polo, Santiago, Navarro y Alí, 2016), que podrían dar mejores luces para comprender el comportamiento del síndrome y sus efectos en la actividad académica estudiantil.

\section{Referencias}

Bandura, A. (1989). Social cognitive theory. In R. Vasta (Ed.), Annals of Child Development, 6. Six theories of child development (pp.1-60). Greenwich, CT: JAI Press.

Barco, V., Miranda, Y., Herrera, D., \& Álvarez, Z. (2008). El síndrome de Burnout en enfermeros básicos del segundo año de la carrera. Revista Cubana Enfermería, 24(3-4), 0-0.

Barraza, A., Carrasco, R., y Arreola M., G. (2009). Burnout estudiantil. Un estudio exploratorio. En A. Barraza, D. Gutiérrez y D. I. Ceniceros (Coords.): Alumnos y profesores en perspectiva (pp.68-84). Durango (México): Universidad Pedagógica de Durango. 
Barraza, A., Ortega, F., \& Ortega, M. (2013). Síndrome de Burnout en alumnos de Doctorado en Educación de la Ciudad de Durango. Enseñanza e Investigación en Psicología, 18(1), 85-94.

Bittar, M. (2008). Burnout y estilos de personalidad en estudiantes universitarios. Recuperado de http://fci. uib.es/digitalAssets/177/177915_2.pdf

Borda, M., Navarro, E., Aun, E., Berdejo, H., Racedo, K. \& Ruiz, J. (2007). Síndrome de Burnout en estudiantes de internado del Hospital Universidad del Norte. Salud Uninorte, 23(1), 43-51.

Bresó, E., \& Salanova, M. (2001). Satisfacción y compromiso académico en estudiantes universitarios. Recuperado de http://repositori.uji.es/xmlui/bitstream/handle/10234/79721/forum_2001_24. pdf?sequence $=1$

Caballero, C., Abello, R., \& Palacio, J. (2006). Burnout, engagement y rendimiento académico entre estudiantes universitarios que trabajan y aquellos que no trabajan. Revista Psicogente, 9(16), 11-27.

Caballero, C., Abello, R., \& Palacio, J. (2007). Relación del Burnout y rendimiento académico con la satisfacción frente a los estudios en estudiantes universitarios. Avances en Psicología Latinoamericana, 25(2), 98-111.

Caballero, C., Hederich, C., \& Palacio, J. (2010). El Burnout académico: delimitación del síndrome y factores asociados con su aparición. Revista Latinoamericana de Psicología, 42(1), 131-146.

Caballero, C., Hederich, C., \& Palacio, J. (2012). Sindrome de Burnout: Prevalencia y factores asociados en estudiantes universitarios del área de la Salud, de la ciudad de Barranquilla (Tesis no publicada).

Carlotto, M., Goncalves, S., \& Borges, A. (2005). Predictores del síndrome en estudiantes de un curso técnico de Enfermería. Diversitas Perspectiva en Psicología, 1(002), 195-204.

Cherniss, C. (1993). Role of professional self-efficacy in the etiology and amelioration of Burnout. En Schaufeli, W. B. (Ed.), Maslach, Ch. (Ed.). Professional Burnout: Recent developments in theory and research. Series in applied psychology: Social issues and questions (pp.135-149). Washington, DC.: EE. UU.

Cordes, C.L., \& Gougherty, T.W. (1993). A review and an integration of research on job Burnout. Academy of Management Review, 18, 621-656.

Deary, I., Watson, R., \& Houston, R. (2003). A longitudinal cohort study of Burnout and attrition in nursing students. Journal of advanced nursing, 43(1), 71-81.

Drybye, L., Thomas, M., Huntington, J., Lawson, K., Novotny, P., Sloan, J. \& Shanafelt, T. (2006). Personal life events and medical student Burnout: A multicenter study. Academic Medicine, 81(4), 374 384.

Ferrel, R., Pedraza, C., \& Rubio, B. (2010). El síndrome de quemarse en el trabajo (Burnout) en docentes universitarios. Duazary, 7(1), 15-28.

Garcés de Los Fayos, E. J. (1995). Burnout en niños y adolescentes: un nuevo síndrome en psicopatología infantil. Psicothema, 7(1), 33-40. 
Gil-Monte, P., \& Peiró, J. (1999). Perspectivas teóricas y modelos interpretativos para el estudio del síndrome de quemarse por el trabajo. Anales de Psicología, 15(2), 261-268.

Gil-Monte, P. (2001). El síndrome de quemarse por el trabajo (síndrome de Burnout): aproximaciones teóricas para su explicación y recomendaciones para la intervención. PsicoPediaHoy, 3(5).

Hernández, R., Fernández, C., \& Baptista, P. (2010). Metodología de la Investigación. Quinta edición. México: McGraw-Hill/Interamericana.

Lee, R.T., \& Ashforth, B.E. (1996). A meta-analytic examination of the correlates of the three dimensions of job Burnout. Journal of Applied Psychology, 81, 123-133.

Leiter, M.P. (1992). Burnout as a crisis in self-efficacy: Conceptual and practical implications. Work y Stress, 6, 107-115.

Martínez, I., \& Márquez, A. (2005). Burnout en estudiantes universitarios de España y Portugal y su relación con variables académicas. Aletheia, 21, 21-30.

Martínez, I., \& Salanova, M. (2003). Niveles de Burnout $\mathrm{y}$ engagement en estudiantes universitarios. Relación con el desempeño y desarrollo profesional. Revista de Educación, 3(3b), 61-384.

Maslach, Ch., \& Jackson, S.E. (1981). The measurement of experienced Burnout. Journal of Occupational Behaviour, 2, 99-113.
Ortega, C., \& López, F. (2004). El Burnout o síndrome de estar quemado en profesionales sanitarios: revisión y perspectiva 1 . International journal of clinical and Health Psychology, 4(001), 137-160.

Polo, J. D., Santiago, V., Navarro, M. C., \& Alí, A. (2016). Creencias irracionales, síndrome de Burnout y adicción al trabajo en las organizaciones. Psicogente, 19(35), 148-160.

Preciado, M., \& Vázquez, J. (2010). Perfil de estrés y síndrome de Burnout en estudiantes mexicanos de Odontología de una universidad pública. Revista Chilena de Neuro-Psiquiatría, 48(1), 11-19.

Salanova, M., \& Schaufeli, W. (2004). El engagement de los empleados: un reto emergente para la dirección de los recursos humanos. Rev. Estudios Financieros, (261), 109-138.

Salanova, M., Bresó, E., \& Schaufeli, W.B. (2005) Hacia un modelo espiral de las creencias de eficacia en el estudio del Burnout y engagement. Ansiedad y Estrés, 11, 215-231.

Salanova, M., Llorens, S., Cifre, E., Martínez, I., \& Schaufeli, W.B. (2003). Perceived collective efficacy, subjective well-being and task performance among electronic work groups: An experimental study. Small Groups Research, 34, 43-73.

Salanova, M., Martínez, I., Bresó, E., Llorens, S., \& Grau, R. (2005). Bienestar psicológico en estudiantes universitarios: facilitadores y obstaculizadores del desempeño académico. Anales de Psicología, 21(1), 170-180. 
Santen, S.A., Holt, D.B., Kemp, J.D., \& Hemphill, R.R. (2010). Burnout in medical students: examining the prevalence and associated factors. South Med J, 103(8), 758-63.

Schaufeli, W., Martínez, I., Márquez, A., Salanova, M., $\&$ Bakker, A. (2002). Burnout and engagement in universitary student: A cross-national study. Journal of cross-cultural psychology, 33(5), 464-481.

Schaufeli, W.B., Salanova, M., González-Romá, V., \& Bakker, A. (2002). The measurement of Burnout and engagement: A confirmatory factor analytic approach. Journal of Happiness Studies, 3, 71-92.
Shirom, A. (1989). Burnout in work organizations. En C. L. Cooper, y I. Robertson (Eds.), International review of industrial and organization psychology (pp. 25-48). Chichester, U.K.: Wiley.

Silvia, R. (2009). Validez y confiabilidad de los instrumentos de recolección de datos. Recuperado de https:// www.slideshare.net/rosilfer/validez-confiabilidad 